\title{
Surgical treatment of multi-valve heart disease associated with infective endocarditis
}

\author{
Hamidullah A. Abdumadzhidov, Hayrullah J. Buranov, Ilkhom K. Huzhakulov, Ikrom I.Mirhodzhaev \\ Republican Specialized Center of Surgery named after academician V. Vahidov, Tashkent, \\ Uzbekistan
}

\begin{abstract}
Objective: The aim of the study was to analyze our own experience of diagnosis, choice of tactics and execution of multi-valve surgical correction of heart defects in infective endocarditis (IE).

Methods: We retrospectively analyzed data and clinical results of 156 patients with infective endocarditis who underwent the cardiac surgery in our clinic. Among them, 85 were men (56.5\%), and women - 71 (45.5\%). Age of our patients ranged from 12 to 68 (mean 32.76 (1.6)) years. The patients were divided into 2 groups: group 1, 89 (57.4\%) patients who underwent a complex of developed by the author's antibiotic therapy, surgical treatment and preventive measures and 2-group 67 (42.6\%) patients who underwent the traditional surgical treatment scheme. We analyzed electrocardiography, chest X-Ray, transthoracic echocardiography, transesophageal echocardiography, cardiac catheterization and coronary angiography and blood culture analysis.

Results: Intraoperative treatment - preventive measures (TPM) were as follows; mechanical and chemical sanitation of the infected area of the heart; implantation of valves with antibacterial properties; hyperthermic perfusion; antimicrobial therapy, including anti-fungal agents. Application of the above measures reduced mortality in the study group to $5.1 \%$, in the control group - $9.3 \%$. In dynamics, mortality declined to $3.9 \%$ in the study group.

Conclusion: Our results of surgical treatment of multi-valve heart defects with infective endocarditis showed the efficacy of developed complex preoperative and intraoperative measures in surgical treatment of IE. This new treatment approach is associated with significant improvement of left ventricular function and low mortality rate.
\end{abstract}

Key words: multi-valve acquired heart diseases, complications of infective endocarditis

(Heart-Vessels and Transplantation 2017; 1: 39-42. doi 10.24969/hvt.2017.24 )

\section{Introduction}

Infective endocarditis (IE), being quite common etiological factor in the development of multi-valve heart defects, has a number of features in the diagnosis, definition of surgical tactics and surgery itself (1-4).

The valvular IE is a septic disease caused by pathogens or conditionally pathogenic microflora, which is the morphological substrate of polypousdestructive ulcerative endocarditis, affecting heart valve and manifesting as acute valvular insufficiency and systemic embolic events. Vegetations on heart valves are the source for embolic complications. Vegetation is composed of fibrin overlay, blood clots, blood cells, damaged heart tissue and microorganisms. The incidence of IE in Russia varies between 2 - 6.5 cases per 100,000 of population (1). Incidence of IE varies between $2 \%-18 \%$ affecting population of 20 to 50 years old, the employed adult population (3).

Address for Correspondence: Hamidullah A.Abdumadzhidov, Department of Reconstructive Surgery

of acquired heart defects JSC Republican Specialized Surgery Center named after academician V.Vahidov, JSC RSCS, 100115 Street, Kichik Halka Yuli, 10, Tashkent, Uzbekistan. Email:

hamidulla06@mail.ru

Received: 15.12.2016 Revised: 23.04.2017 Accepted: 15.05.2017

Copyright $@ 2017$ Heart Vessels and Transplantation 
There is a practice in cardiology to attempt of conservative treatment of infective endocarditis. However, according to some authors, the mortality rate in the conservative treatment is $50-90 \%$ (5). The effectiveness of surgical treatment over medical treatment is obviously clear. However, optimal early and late results of surgical treatment of multi-valve infective endocarditis are largely dependent on the correct definition of surgical tactics and with reduction of mortality to $8.3-11.2 \%$ (4-10). Thus, apparent urgency of the chosen topic on development the most efficient strategy in reduction of mortality the message is not in doubt.

In this article, we decided to discuss our own experience of diagnosis, choice of tactics and execution of multi-valve surgical correction of heart defects in infective endocarditis.

\section{Methods \\ Study design and population}

The population of this retrospective analysis included 156 patients with multi-valve heart disease and infective endocarditis operated in our hospital, of whom 85 were men (54.5\%), and women - 71 (45.5\%). The age of our patients ranged from 12 to 68 (mean 32.76(1.6)) years. Diagnosis was based on the classification criteria of Durack et al. (9).

The patients were divided into 2 groups: group 1 included 89 (57.4\%) patients who underwent a comprehensive anti-bacterial treatment, surgery and preventive measures according to the complex of IE treatment measures developed by our group; group 2 - consisted of 67 (42.6\%) patients who underwent the traditional scheme of prevention and surgical treatment.

The IE treatment protocol:

Taking in account the following features valve infective endocarditis (IE): high frequency of systemic embolism; high mortality in the treatment of IE; the dilemma of the optimal operation period; imperfect system of prevention; aspects of the antimicrobial therapy - we developed in our department comprehensive measures for the prevention and treatment of IE in these patients. They have the following objectives:
1.The elimination of infective focus

2.Hemodynamic wrinkle correction

3. Reliable fixation of the prosthesis

4.Prevention of relapse of IE

Intraoperative treatment and preventive measures (LSM) were as follows:

1. Mechanical and chemical sanitation of infected heart area

2. Implantation of valves with antibacterial properties

3. Hyperthermic perfusion

4. The antimicrobial therapy, including anti-fungal agents.

\section{Clinical examinations}

All patients underwent clinical examinations, electrocardiography (ECG), chest X-ray, transthoracic echocardiography (TTE) and blood culture analysis. Transesophageal echocardiography (TEE) was performed in $40.5 \%$ of patients, coronary angiography and heart catheterization in $12.65 \%$ of patients, histology analysis with light electron microscopy (SEM) in $47.5 \%$ of the operated patients.

\section{Surgery}

Surgical treatment consisted of an "open" correction of affected valves, with carrying out complex measures to eliminate intracardiac foci of infection and prevention of postoperative septic complications. All patients underwent surgery with cardiopulmonary bypass ( $\mathrm{CPB}$ ) and cardioplegia (CP) with conventional protocol of operation in group 2 of patients and protocol of surgical correction developed in our department in group 1 patients (see above).

\section{Statistical analysis}

Statistical analysis of the result was carried out using Microsoft Office Excel 2007 with Statistical Version 6.0 package. Continuous variables are presented as mean and standard error, and categorical data as percentages. Comparison of continuous variables between groups was performed using parametric Student's ( $t$ ) test and Chi-square test was used for comparison of categorical variables. The degree of accuracy was determined at the level of significance $p<0.05$. 


\section{Results and Discussion}

The surgical treatment of multivalvular heart disease with infective endocarditis is a challenging issue. Application of developed by our department complex of medical, surgical and preventive measures aimed at improving significantly the immediate and long-term results of our operations on affected heart valves.

The etiology of infective endocarditis accompanying valvular heart disease in our patients included rheumatic heart valve disease in $62 \%$ of patients, infective endocarditis - $16 \%$, degenerative valvular disease $-13 \%$, and congenital heart disease in $9 \%$ of patients. In our series, there were no patients with infective endocarditis with addiction to substance use. Types of operations performed in our patients are provided in Table 1 . The most commonly performed surgery was mitral valve replacement (MVR) plus aortic valve replacement (AVR) with tricuspid valve (TV) repair. Other types of operations included: mitral valve (MV) repair, aortic valve replacement (AVR), tricuspid valve open commissurotomy (OTC), tricuspid valve replacement (TVR) and mitral valve replacement (MVR). We also used left atrial thrombectomy of in combination with other types of surgery.

\begin{tabular}{|l|c|c|}
\hline \multicolumn{3}{|l|}{ Table 1. The types of surgery performed in patients with multi-valve heart defects with infective endocarditis } \\
\hline Type of surgery & $\mathbf{n}$ & $\%$ \\
\hline MVR+AVR with TV repair & 78 & 50 \\
\hline TV repair, MV repair, and AV repair & 26 & 16.7 \\
\hline MVR with TV repair and AV repair & 21 & 13.4 \\
\hline AVR with TV repair and MV repair & 7 & 4.5 \\
\hline MVR with TV repair & 6 & 3.8 \\
\hline MVR+AVR with OTC & 4 & 2.5 \\
\hline MVR, TVR, AVR & 2 & 1.3 \\
\hline MVR, TVR & 2 & 1.3 \\
\hline Other operations & 10 & 6.4 \\
\hline $\begin{array}{l}\text { AV - aortic valve, AVR -aortic valve replacement, MV - mitral valve, MVR - mitral valve replacement, OTC - open } \\
\text { tricuspid commissurotomy, TV - tricuspid valve, TVR - tricuspid valve replacement }\end{array}$ \\
\hline
\end{tabular}

Use of specially produced artificial mechanical heart valves can prevent further development or activation of the infection. Antibiotic cuff impregnated in artificial prosthesis retains its activity for 2 weeks, which reduces the recurrence of IE (8). In addition, we used hyperthermic perfusion in these patients, targeting micro- and macro-organisms, as following: keeping temperature within $38.5-39^{\circ} \mathrm{C}$ for $15-20 \mathrm{~min}$ after removal of aortic clamp. This, results in "impact on the macro-organism": restoring the patient immunobiological properties (7); and "the impact on the micro-organism" - intensifying pathogen metabolism and enhancing effectiveness of antibiotics (6).
Laboratory and microbiological tests are of particular importance to guide antibioticotherapy. Table 2 shows the results of microbiological tests, where in contrast to literature data: in $5 \%$ of all patients, we identified fungal lesions of the heart valves - Candida spp. The blood culture was positive in $39.1 \%$ of patients, in $8.8 \%$ of the cases we detected multidrugresistant Gram-negative flora, in 7\% - Staphylococus aureus, and in $6.3 \%$ of cases - Pseudomonas aeruginosa. In remaining $60.9 \%$ of patients, the blood culture was negative due to wide-spectrum antibiotic therapy used on pre-hospital state. According to above-mentioned results, we used antifungal therapy in addition to antibiotics. 


\begin{tabular}{|l|l|}
\hline Table 2. Microbiological examination \\
\hline Microorganisms & Frequency allocation, \% \\
\hline Gram (-) negative bacillus & 8.8 \\
\hline Gram (+) positive bacillus & 2.5 \\
\hline Staphylococcus aureus & 7 \\
\hline Pseudomonas aeruginosa & 6.3 \\
\hline Streptococcus epidermidis & 5 \\
\hline Candida spp. & 5 \\
\hline Klebsiella pneumoniae & 4 \\
\hline Other & 0.5 \\
\hline Total & 39.1 \\
\hline No identified & 60.9 \\
\hline
\end{tabular}

Echocardiography had a particular importance in adequate definition and choice of surgical tactics for our patients. Transthoracic 2-dimensional and Doppler echocardiography data in addition to the clinical criteria were the basis of indications for surgical correction of multi-valvular defects with IE, preoperative echocardiography data are presented in
Table 3. Surgical tactics of the operation were identified taking in account the peculiarities of defect anatomy, disease complications, and presence of comorbidities. At intraoperative stage TEE was carried out aiming at refining the tactics of surgical correction and evaluation of the adequacy of the operation.

\section{Table 3. Preoperative echocardiography data}

\begin{tabular}{|c|l|l|c|c|}
\hline \multirow{2}{*}{ LV parameters } & Group 1 & Group 2 & Overall & p \\
\cline { 2 - 5 } & & & & \\
\hline EDD, $\mathbf{~ l ~}$ & $5.88(0.15)$ & $6.16(0.18)$ & $6.01(0.12)$ & $>0.05$ \\
\hline EDV, ml & $181.8(9.81)$ & $194.55(13.4)$ & $187.3(8.01)$ & $>0.05$ \\
\hline ESD, $\mathbf{~ m l}$ & $3.88(0.12)$ & $7.05(1.65)$ & $5.3(0.76)$ & $<0.05$ \\
\hline ESV, $\mathbf{m l}$ & $83.64(12.1)$ & $81.86(6.82)$ & $82.8(7.44)$ & $>0.05$ \\
\hline SV, $\mathbf{~ m l}$ & $111.24(5.9)$ & $116.45(7.5)$ & $113.5(4.69)$ & $>0.05$ \\
\hline EF, \% & $63.21(1.96)$ & $60.3(1.19)$ & $61.83(1.23)$ & $<0.05$ \\
\hline
\end{tabular}

Data are presented as Mean (SE)

EDD - end-diastolic dimension, EDV - end-diastolic volume, EF-ejection fraction, ESD - end -systolic dimension, ESV - end-systolic volume, LV-left ventricle, SV-stroke volume

Indication for surgical treatment in our patients, in most cases, was progressive heart failure. As can be seen from the Table 4, among life-threatening complications of IE requiring implementation of sometimes urgent indications for "health reasons" surgical treatment, the main was progressive heart failure, which prevailed by the overwhelming number of cases. At the same time, we are sure that lower inflammatory activity may be achieved using a complex of medical and antibiotic therapy. The combination of the preoperative preparation, using the developed therapeutic and preventive measures, subsequently, was the basis to decrease mortality in the short and long periods of observation. 


\begin{tabular}{|l|c|c|}
\hline \multicolumn{2}{|c|}{ Table 4. Indications for the implementation of multi-valve defect surgery with infective endocarditis } \\
\hline Indications for surgery & Number & \% \\
\hline Progressive heart failure & 96 & 12.4 \\
\hline $\begin{array}{l}\text { The ineffectiveness of antibiotic } \\
\text { therapy }\end{array}$ & 20 & 8.9 \\
\hline Floating vegetation & 14 & 10.9 \\
\hline Embolism & 17 & 5.9 \\
\hline Abscess & 9 & 100 \\
\hline Total operated & 156 & \\
\hline
\end{tabular}

Analysis of the nearest results of our operations in the two groups showed mortality rate of $5.1 \%$ in the first group, and $9.3 \%$ in the second group. Thus, the overall hospital mortality was $6.9 \%$. With the development of diagnostic criteria for multi-valve defect with IE, choosing the right surgical technique and the use of therapeutic and preventive measures developed in the department, we managed to reduce the rate of hospital mortality and improve cardiac function. We observed in a 3-year follow-up period reduction of cardiac chambers and volumes size (Table 5) and significant reduction of hospital mortality, as compared with previous years (from $33 \%$ in 2000 2001 to $3.9 \%$ in $2011-2015$ periods) (Fig. 1).

\begin{tabular}{|c|c|c|c|}
\hline Echocardiographic data & \multicolumn{3}{|c|}{ The periods of observation } \\
\hline Group 1 & Before surgery & At discharge & 3 years later \\
\hline EDD, $\mathrm{ml}$ & $5.88(0.15)$ & $5.03(0.13)$ & $4.65(0.16)$ \\
\hline EDV, $\mathrm{ml}$ & $181.8(9.81)$ & $133.63(8.12)$ & $105.4(7.43)$ \\
\hline ESD, ml & $3.88(0.12)$ & $3.67(0.11)$ & $3.3(0.21)$ \\
\hline $\mathrm{ESV}, \mathrm{ml}$ & $83.64(12.12)$ & $67.58(6.56)$ & $45.4(5.76)$ \\
\hline $\mathrm{SV}, \mathrm{ml}$ & $111.24(5.97)$ & $65.8(2.97)$ & $60.01(3.32)$ \\
\hline$E F, \%$ & $63.21(1.96)$ & $51.05(1.48)$ & $61.45(2.5)$ \\
\hline$p$ & \multicolumn{3}{|c|}{$<0.03$} \\
\hline \multicolumn{4}{|l|}{ Group 2} \\
\hline EDD, $\mathrm{ml}$ & $6.16(0.18)$ & $5.34(0.17)$ & $5.14(0.15)$ \\
\hline EDV, ml & 194.5 (13.39) & $147.13(11.21)$ & $125.71(8.07)$ \\
\hline ESD, ml & $5.25(1.01)$ & $5.1(1.22)$ & $3.3(0.21)$ \\
\hline ESV, ml & $81.86(6.82)$ & $73.16(7.05)$ & $47.93(5.91)$ \\
\hline $\mathrm{sV}, \mathrm{ml}$ & $116.45(7.55)$ & $73.63(4.95)$ & $77.6(6.06)$ \\
\hline$E F, \%$ & $60.0(1.19)$ & $52.34(1.57)$ & $58.36(3.16)$ \\
\hline p & \multicolumn{3}{|c|}{$<0.05$} \\
\hline
\end{tabular}




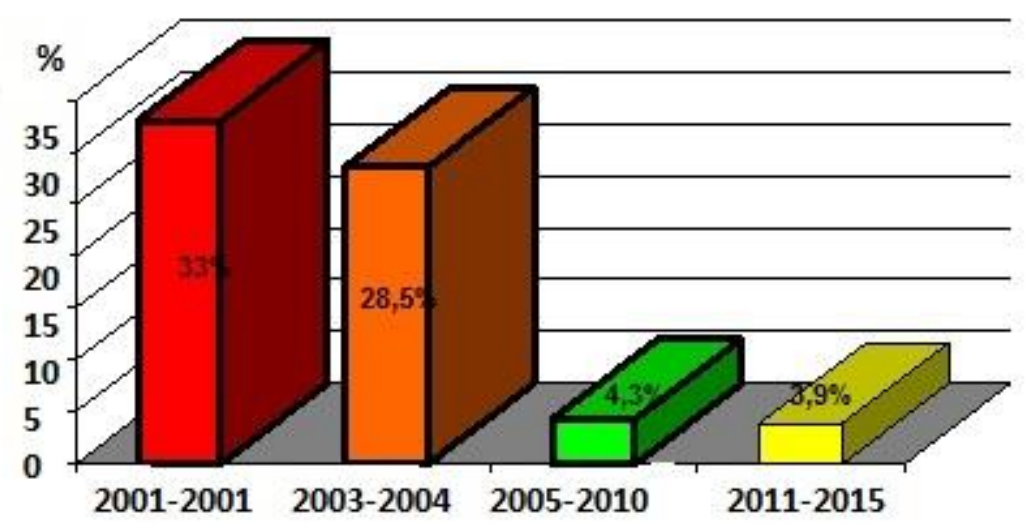

Figure 1. Dynamics of hospital mortality

\section{Study limitations}

Our study has certain limitations as being retrospective in design and relatively small numbers of patients. Further prospective studies need to be undertaken to clarify the usefulness of our approach of treatment of IE associated with multi-valvular heart defects.

\section{Conclusion}

Our results of surgical treatment of multi-valve heart defects with IE showed the efficacy of developed complex preoperative and intraoperative measures in surgical treatment of IE. This new treatment approach is associated with significant improvement of left ventricular function and low mortality rate.

Peer-review: Internal and external

Conflict of interest: None to declare

Authorship: H.A.A., H.J.B, I.K.H., I.I.M. equally contributed to the study and preparation of manuscript Acknowledgement and funding: None to declare Informed consent for procedures and treatment was obtained from all patients as a part of center policy (retrospective study)

\section{References}

1. Bokeria LA, Stupakov IN, Samorodskaya IV. The incidence and the need for cardiac treatment methods in the aspect of epidemiological analysis. Thorac Cardiovasc Surg 2006; 1: 4-7.

2. Bokeria LA, Stupakov IN, Gudkov RG. Surgery of acquired heart disease in Russia. XIIIth All-Russian Congress of Cardiovascular Surgeons. Moscow 25-28 November 2007. Bulletin NTSSSH by academician Bakulev RAMS 2007; 8: 36.

3. Zheltovsky YV, Walk EV, Podkamennaya VA. The use of immobilized proteolytic enzymes in the surgical treatment of active infective endocarditis. Pathol Circul Card Surg 2006; 4: 92-6.

4. Nishimura RA, Carabello BA, Faxon DP, Freed MD, Lytle BW, O'Gara PT, et al. ACC / AHA 2008 guideline update on valvular heart disease: focused update on infective endocarditis. A report of the ACC / AHA Task Force on Practice Guidelines; endorsed by the Society of Cardiovascular Anesthesiologists, Society for Cardiovascular Angiography and Interventions, and Society of Thoracic Surgeons. Circulation 2008; 118: 887-96.

5. Polyakov VP, Shorohov SE. Infective valve endocarditis (modern view on the problem) Samara, 2004. $137 \mathrm{p}$.

6. Prikhodko VP. Features of surgical approach in the treatment of primary and secondary infective endocarditis. Chelyabinsk. 2003. (Dissertation DMSc).

7. Rudenko AV, Screamers AA. Clinical manifestations and diagnosis of infective endocarditis. Cardiovasc Surg (Kiev) 2004; 1: 96-104.

8. Actis D, Cavaglia M, Poletti G. Impregnation of sewing ring with antibiotics to avoid prosthetic valve endocarditis. J Thorac Cardiovasc Surg 2004; 114: 1456.

9. Durack DT, Bright DK, Lukes AS. Duke Endocarditis Service. New criteria for diagnosis of infective endocarditis: utilization of specific echocardiographic findings. Am J Med 2004; 96: 200-9.

10. Horstkotte $D$, Follath $F$, Gutschik $E$, Lengyel $M$, Oto A, Pavie A, et al. Guidelines on prevention, diagnosis and treatment of infective endocarditis executive summary; the task force on infective endocarditis of the European society of cardiology. Executive Summary. Eur Heart J 2004; 25: 267-76. 